\title{
Intravitreal bevacizumab associated with grid laser photocoagulation in macular edema secondary to branch retinal vein occlusion
}

\author{
Angel Salinas-Alamán, Javier Zarranz-Ventura, Jose M. Caire González-Jauregui, \\ Luis M. Sádaba-Echarri, Jesús Barrio-Barrio, Alfredo García-Layana \\ Department of Ophthalmology, Clínica Universidad de Navarra, Pamplona, Navarra - Spain
}

\begin{abstract}
PURPOSE. To evaluate intravitreal bevacizumab (IVB) combined with grid laser photocoagulation in macular edema (ME) secondary to branch retinal vein occlusion (BRVO).

METHODs. Eight eyes (8 patients) with ME associated with BRVO with at least 3 months of evolution since symptom onset were included. All subjects underwent measurement of best-corrected visual acuity (BCVA) and imaging with spectral domain optical coherence tomography (SD-OCT) at baseline and 1, 3, 6, and 12 months. Intravitreal bevacizumab was administered at baseline and macular grid laser photocoagulation 1 month later. During follow-up, additional IVB was administered based on physician discretion if persistent or recurrent intraretinal fluid (cysts) was observed on SD-OCT. The mean BCVA and SD-OCT central subfield thickness (CST) values were determined at each time point. Fisher exact test was performed to assess differences between baseline and post-treatment BCVA and SD-OCT measurements.

RESULTS. The mean baseline BCVA was $0.28 \pm 0.14$ (mean $\pm S D$ ), and the mean CST was $479 \pm 137 \mu \mathrm{m}$. The mean BCVAs at $1,3,6$, and 12 months were $0.47 \pm 0.18(p=0.031), 0.56 \pm 0.50(p=0.031), 0.65 \pm 0.60$ $(p=0.008)$, and $0.66 \pm 0.65(p=0.016)$, respectively. The mean CST values at $1,3,6$, and 12 months were $295 \pm 60 \mu \mathrm{m}(p=0.008), 333 \pm 114 \mu \mathrm{m}(p=0.070), 339 \pm 80 \mu \mathrm{m}(p=0.008)$, and $335 \pm 109 \mu \mathrm{m}(p=0.008)$. A mean 2.13 injections were administered; the second injection was administered a mean of 2.71 months after baseline.

ConcLusions. Combined treatment with IVB and macular grid photocoagulation provided good results and may be considered as an alternative therapy for ME in BRVO. Further studies are needed to assess these preliminary results.
\end{abstract}

KEY WoRDs. Avastin, Bevacizumab, Branch retinal vein occlusion, Grid laser, Laser photocoagulation, Macular edema

Accepted: November 2, 2010

\section{INTRODUCTION}

Branch retinal vein occlusion (BRVO) is the second most common retinal sight-threatening vascular disorder, the frequency of which is surpassed only by diabetic retinopathy (1-3). The occlusion usually develops in an arteriovenous nicking, where both vessels share the adventitia. The visual acuity (VA) decreases because of concurrent retinal hemorrhages, macular ischemia, and macular edema (ME), which is the most frequent factor that affects vision in the short, intermediate, and especially long term.

Several therapeutic approaches have been proposed for BRVO ranging from isovolemic hemodilution to vitrectomy (4-10). Since publication of the Branch Vein Occlusion Study Group results in 1984, in which investigators found a significant mean improvement of 1.33 lines of vision com- 


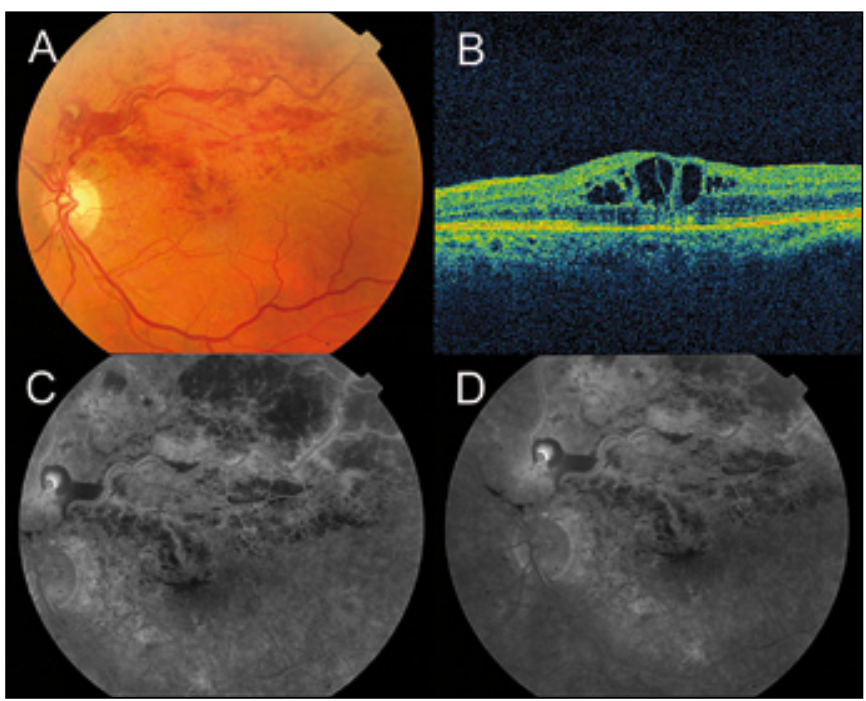

Fig. 1 - Case 3. (A) Baseline color fundus photograph. Multiple hemorrhages and venous tortuosity are seen along the occluded vein area. The foveal area appears to be affected. A macroaneurysm is also present with associated preretinal hemorrhage. (B) Spectral domain optical coherence tomography (SD-OCT) at baseline shows macular edema with intraretinal cysts in the inner retinal layers. (C, D) Baseline intermediate (C) and late-phase (D) fluorescein angiography angiograms show macular edema in the fundus and the foveal center, with intraretinal cysts in this area assessed by SD-OCT. The best-corrected visual acuity has decreased to 0.1 .

pared with 0.23 line in controls after 3 years of follow-up with a level of evidence $A$, grid laser photocoagulation has been considered the gold standard for treating $\mathrm{ME}$ in patients with BRVO (6). Recently, alternative therapies, i.e., intravitreal injections of corticosteroids, anti-vascular endothelial growth factor (VEGF) drugs, or new devices for extended drug release such as dexamethasone implants, have been proposed, sometimes in combination treatment protocols that have not provided definitive medium- and long-term results. We propose a previously unreported alternative treatment based on combination treatment with intravitreal antiangiogenic therapy and macular grid laser photocoagulation of the affected areas in a series of patients with ME associated with BRVO.

\section{METHODS}

Eight eyes of 8 patients with ME secondary to BRVO and decreased VA were prospectively included between May and November 2008. Every patient had had at least 3 months of evolution since symptom onset, described as

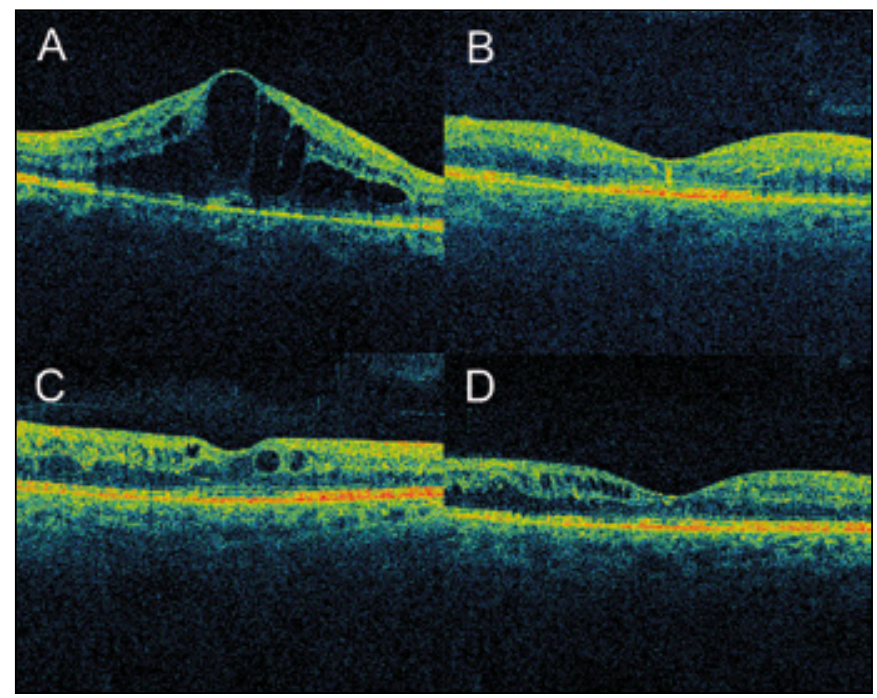

Fig. 2 - Case 5. (A) Baseline spectral domain optical coherence tomography. The best-corrected visual acuity (BCVA) is 0.3. (B-D) Spectral domain optical coherence tomography images at 1, 3, and 12 months after treatment. The first injection resolved the swelling of the macular area and grid laser was performed 1 month later. Spectral domain optical coherence tomography at the third month visit shows fluid recurrence. Another dose of bevacizumab is required, and 12 months after the first injection the retina appears flat with isolated hyporeflective foci, no signs of swelling, and a good visual outcome (BCVA 0.9).

metamorphopsia or decreased VA ranging from 0.1 to 0.5 assessed with 6-meter Snellen charts. All patients had multiple hemorrhages in the affected BRVO areas and ME assessed by spectral domain optical coherence tomography (SD-OCT) (Fig. 1). The Ethics Committee of our hospital approved the study, which was conducted in accordance with the tenets of the Declaration of Helsinki. All patients provided informed consent before entering the study.

At the first visit, all patients underwent a standard ophthalmic evaluation of best-corrected visual acuity (BCVA) with 6-meter Snellen charts and SD-OCT imaging with a macular cube $256 \times 128$ scan protocol (Cirrus HD-OCT ${ }^{\circledR}$, Carl Zeiss Meditec, Dublin, CA, USA). The tomographic parameter analyzed in this study was the central subfield thickness (CST), which was measured at baseline and 1, 3, 6, and 12 months after treatment (Fig. 2). The main outcome measures were BCVA and CST for all visits during the study period.

Intravitreal injections of $1.25 \mathrm{mg}$ of bevacizumab (Avastin ${ }^{\circledR}$, Genentech Inc., South San Francisco, CA, USA) in a 0.05$\mathrm{mL}$ volume were administered in the clinic. After instilling $0.5 \%$ proparacaine hydrochloride topical eyedrops 3 times every 5 minutes for topical anesthesia, the eye was irrigat- 
ed with $5 \%$ povidone iodine, opened using a lid retractor, and the drug was injected through the pars plana $3.0 \mathrm{~mm}$ posterior to the limbus in pseudophakic eyes and $3.5 \mathrm{~mm}$ posterior to the limbus in phakic eyes using a 30-gauge needle. After every intravitreal injection, an ophthalmic solution of topical ofloxacin (Exocin ${ }^{\circledR}$, Allergan Pharmaceuticals Ltd., Ireland) was administered 4 times daily for 1 week. Intravitreal bevacizumab (IVB) injections were administered at baseline in all patients; reinjections were administered if persistent or recurrent intraretinal fluid (cysts) was observed in SD-OCT.

Laser application was performed with a pan-funduscopic TransEquator $^{\circledR}$ lens (Volk Optical Inc., Mentor, OH, USA) (spot diameter, 50 um; exposure time, $200 \mathrm{~ms}$; power, 80$100 \mathrm{~mW}$ ) until soft whitening of the retina became apparent, according to physician discretion. Grid laser photocoagulation was performed over the area of ME. Laser spots were not applied a minimum of $500 \mu \mathrm{m}$ from the foveal center. Laser was applied 1 month after the first IVB injection in all cases. One patient (case 2) underwent sectorial laser photocoagulation at baseline due to peripheral ischemia observed on fluorescein angiography. No additional macular grid laser photocoagulation was performed during follow-up in any case.

Statistical analysis was performed with SPSS 15.0 software (SPSS Inc., Chicago, IL, USA). Fisher exact test was used to assess baseline and post-treatment differences in VA and central subfield thickness (CST) at all time points. The significance level was $p \leq 0.05$.

\section{RESULTS}

The mean patient age was $71.6 \pm 7.68$ (mean \pm standard deviation) years. The demographic features and individual patient data are shown in Table I. The mean baseline BCVA

TABLE I - DEMOGRAPHICS AND INDIVIDUAL FEATURES OF INCLUDED PATIENTS

\begin{tabular}{|c|c|c|c|c|c|c|c|c|c|c|c|c|c|c|}
\hline \multirow[t]{2}{*}{ Case } & \multirow{2}{*}{$\begin{array}{c}\text { Age, } \\
y\end{array}$} & \multirow[t]{2}{*}{ Sex } & \multicolumn{5}{|c|}{ Best-corrected visual acuity } & \multicolumn{5}{|c|}{ Central subfield thickness } & \multirow[t]{2}{*}{ Doses } & \multirow{2}{*}{$\begin{array}{c}\text { Months } \\
\text { 2nd injection }\end{array}$} \\
\hline & & & Baseline & $1 \mathrm{mo}$ & $3 \mathrm{mo}$ & $6 \mathrm{mo}$ & $12 \mathrm{mo}$ & Baseline & $1 \mathrm{mo}$ & $3 \mathrm{mo}$ & $6 \mathrm{mo}$ & $12 \mathrm{mo}$ & & \\
\hline 1 & 76 & $\mathrm{M}$ & 0.4 & 0.7 & 0.8 & 0.8 & 0.8 & 545 & 190 & 182 & 370 & 305 & 1 & - \\
\hline 2 & 79 & $\mathrm{~F}$ & 0.15 & 0.4 & 0.6 & 0.6 & 0.7 & 494 & 346 & 327 & 304 & 221 & 2 & 3 \\
\hline 3 & 63 & $M$ & 0.10 & 0.4 & 0.4 & 0.4 & 0.5 & 737 & 236 & 362 & 370 & 393 & 2 & 3 \\
\hline 4 & 74 & $\mathrm{~F}$ & 0.4 & 0.6 & 0.4 & 0.8 & 0.8 & 361 & 281 & 359 & 355 & 311 & 2 & 3 \\
\hline 5 & 62 & $\mathrm{~F}$ & 0.3 & 0.7 & 0.9 & 0.9 & 0.9 & 362 & 292 & 253 & 249 & 251 & 2 & 3 \\
\hline 6 & 73 & $M$ & 0.5 & 0.5 & 0.5 & 0.6 & 0.5 & 497 & 371 & 560 & 348 & 461 & 2 & 3 \\
\hline 7 & 64 & $\mathrm{~F}$ & 0.2 & 0.2 & 0.5 & 0.5 & 0.5 & 534 & 341 & 378 & 486 & 515 & 4 & 1 \\
\hline 8 & 82 & $\mathrm{M}$ & 0.2 & 0.3 & 0.4 & 0.6 & 0.6 & 305 & 304 & 247 & 230 & 230 & 2 & 3 \\
\hline Mean & 71.63 & - & 0.28 & 0.47 & 0.56 & 0.65 & 0.66 & 479.38 & 295.13 & 333.50 & 339.00 & 335.88 & 2.13 & 2.71 \\
\hline SD & 7.68 & - & 0.14 & 0.18 & 0.19 & 0.16 & 0.15 & 137.52 & 60.10 & 114.45 & 80.24 & 109.66 & 0.83 & 0.75 \\
\hline
\end{tabular}

Demographics and individual features of patients included in the study. Best-corrected visual acuity and central subfield thickness assessed by spectral domain optical coherence tomography at baseline, month 1, month 3, month 6 , and month 12 post-treatment; total number of intravitreal bevacizumab (IVB) doses per eye and months post-treatment in which 2 nd dose of IVB was performed (if required).

TABLE II - MEAN BEST-CORRECTED VISUAL ACUITY AND MEAN CENTRAL SUBFIELD THICKNESS AT DIFFERENT TIMEPOINTS

\begin{tabular}{|c|c|c|c|c|}
\hline & $\begin{array}{c}\text { Mean best-corrected visual } \\
\text { acuity }\end{array}$ & $\mathbf{p}$ & $\begin{array}{c}\text { Mean central subfield } \\
\text { thickness }\end{array}$ & $\mathbf{p}$ \\
\hline Baseline & 0.28 & & 479.38 & \\
\hline $1 \mathrm{mo}$ & 0.47 & $0.031^{\star}$ & 295.13 & $0.008^{*}$ \\
\hline $3 \mathrm{mo}$ & 0.56 & $0.031^{\star}$ & 333.50 & 0.070 \\
\hline $6 \mathrm{mo}$ & 0.65 & $0.008^{\star}$ & 339.00 & $0.008^{*}$ \\
\hline $12 \mathrm{mo}$ & 0.66 & $0.016^{\star}$ & 335.88 & $0.008^{*}$ \\
\hline
\end{tabular}

Mean best-corrected visual acuity and mean central subfield thickness assessed by spectral domain optical coherence tomography at baseline, month 1 , month 3 , month 6 , and month 12 post-treatment. ${ }^{*} \mathrm{p} \leq 0.05$. 
was $0.28 \pm 0.14$ Snellen, and at $1,3,6$, and 12 months after treatment the BCVA levels were $0.47 \pm 0.18(p=0.031)$, $0.56 \pm 0.50(p=0.031), 0.65 \pm 0.60(p=0.008)$, and $0.66 \pm 0.65$ $(p=0.016)$, respectively (Tab. II). The mean baseline CST was $479 \pm 137 \mu \mathrm{m} ; 1,3,6$, and 12 months after treatment the mean CST values were $295 \pm 60 \mu \mathrm{m}(\mathrm{p}=0.008), 333 \pm 114$ $\mu \mathrm{m}(\mathrm{p}=0.070), 339 \pm 80 \mu \mathrm{m}(\mathrm{p}=0.008)$, and $335 \pm 109 \mu \mathrm{m}$ $(p=0.008)$ (Tab. II). The mean number of injections was $2.13 \pm 0.83$. In 7 patients who required a second injection, the injection was administered a mean of 2.71 months (SD 0.75 ) after the first dose. One patient required 4 injections during follow-up, and another patient did not receive additional treatment.

\section{DISCUSSION}

Intravitreal bevacizumab associated with grid laser photocoagulation decreased the CRT assessed by SD-OCT and improved the VA in patients with ME secondary to BRVO. After the first IVB injection, a decrease in the CRT was observed in all cases associated with an increase in the VA before and after grid laser photocoagulation. We did not observe the same effect with the second injection administered at about month 3 of follow-up, 2 months after grid laser photocoagulation. Although the CRT did not steadily decrease after the second IVB injection, the VA improvement was sustained from the third month through the remainder of the follow-up period. Thus, from the 3-month evaluation, we did not find a good correlation between the VA and the CRT, which agreed with the results of previous studies with a larger number of patients with ME secondary to BRVO (1). However, this unexpected discrepancy suggested that other factors may be involved, i.e., the integrity of the junction between the inner and outer photoreceptor segments on SD-OCT, the volume of intraretinal cysts, or retinal pigment epithelium atrophy. Unfortunately, assessment of the influence of these factors in our results exceeds the limitations of this study.

The treatment of ME secondary to BRVO has been controversial $(2,3)$. Ranging from isovolemic hemodilution (4) to vitrectomy with sheathotomy (5) of the arteriovenous nicking, therapies such as laser grid photocoagulation or intravitreal drug injections have not provided definitive satisfactory, evidence-based results. Since the Branch Vein Occlusion Study Group published its results in 1984, grid laser photocoagulation has been the gold standard treat- ment for ME in patients with BRVO. In the current study, a gain of 2 lines of vision was observed at 8 months in $65 \%$ of patients; that increase was 1.3 lines 3 years after laser application (6). These results have not been clearly surpassed by other treatments in comparably designed studies.

Several authors have considered surgery as a treatment for ME associated with BRVO. Pars plana vitrectomy with posterior hyaloidectomy and sheathotomy of the arteriovenous nicking resolved the ME in different series (7-9). Whereas the technical difficulty of the surgery was the main limitation, other authors have suggested that sheathotomy was unnecessary and showed similar results in a comparative study (10). Nevertheless, the inherent inconvenience of surgery and the limited surgical results have shifted the focus to other less-invasive therapies.

The development of pharmacologic intravitreal treatment has been remarkable in recent years, and these drugs currently are used routinely to treat diverse retinal pathologies. Intravitreal injection of corticosteroids, especially triamcinolone acetonide (TA), has been widely used to treat ME with diverse etiologies, including BRVO. Several authors have reported good short- and intermediate-term VA results with TA (11-15). Unfortunately, these studies had small numbers of patients and frequently lacked a control group for comparison. The findings cannot be considered definitive and larger studies must confirm them. More recently, the Standard Care vs Corticosteroid for Retinal Vein Occlusion (SCORE) study, which included a large number of patients and a 12-month follow-up period, reported that intravitreal injection of TA did not obtain better results than those in the control group treated with grid laser photocoagulation (16). However, other studies have compared TA use combined with grid laser photocoagulation and obtained acceptable results in short series $(17,18)$, but problems related to intravitreal administration, i.e., glaucoma, cataract development, or noninfectious endophthalmitis, and the fact that TA administration is an off-label use, have reduced physician interest in this treatment. To overcome these problems, new corticosteroid delivery devices have been developed. The dexamethasone implant, which was approved recently by the Food and Drug Administration for use in patients with BRVO, has reduced the risk of vision loss and improved the speed and incidence of visual improvement at 6 months compared to sham controls with moderate adverse effects (19-21). Although these results have been promising, they must be ratified in further reports with longer follow-up periods. 
In the last decade, the results achieved with anti-VEGF drugs in age-related macular degeneration and the suggested role of VEGF in the development of ME secondary to vascular pathologies have led to a hypothesis about their effectiveness. Therefore, several studies have been performed to assess ranibizumab (Lucentis ${ }^{\circledR}$, Genentech Inc.), bevacizumab, and pegaptanib (Macugen ${ }^{\circledR}$, Pfizer/ Eyetech Pharmaceutical) on ME in patients with BRVO (22-26). Most studies have been uncontrolled, nonrandomized short case series, and it is impossible to make reliable comparisons among them. However, Campochiaro and the BRAVO Study Group recently reported significant visual improvement with intravitreal ranibizumab compared to sham injection at 6 months, with a lower rate of rescue grid laser than controls and no increased adverse effects (27). The medium- and long-term data of this study will provide important information about its role in clinical practice.

The clinical observation that antiangiogenic drugs, specifically bevacizumab, effectively decreased the ME associated with BRVO led to the current study. The aim of our study was to add a beneficial transient effect over the vascular permeability of IVB to the long-term macular stabilization provided by grid laser photocoagulation. Although the mechanism of this laser-related beneficial effect remains unknown and several hypotheses have been proposed, we consider that the benefit may be due to retinal pigment epithelium stimulation. To our knowledge, this is the first study to propose this sequential combination treatment in a series of patients with ME associated with BRVO. The results obtained were promising, but the small number of patients, the lack of a control group, and the limited 12-month follow-up period does not permit us to draw definitive conclusions. However, the results of this study are a well-founded basis to develop further studies involving a larger number of patients to confirm these results. In conclusion, the findings suggested that combination treatment comprised of IVB and grid laser photocoagulation may be an alternative option for these patients.

Proprietary interest: None.

Address for correspondence:

Javier Zarranz-Ventura, MD

Department of Ophthalmology

Clínica Universidad de Navarra

Avda. Pío XII No 36

31080 Pamplona

Navarra

Spain

jzarranz@unav.es

\section{REFERENCES}

1. Scott IU, VanVeldhuisen PC, Oden NL, et al, SCORE Study Investigator Group. SCORE Study Report 1: baseline association between central retinal thickness and visual acuity in patients with retinal vein occlusion. Ophthalmology 2009; 116: 504-12.

2. Rehak J, Rehak M. Branch retinal vein occlusion: pathogenesis, visual prognosis, and treatment modalities. Curr Eye Res 2008; 33: 111-31.

3. McIntosh RL, Mohamed Q, Saw SM, Wong TY. Interventions for branch retinal vein occlusion: an evidence-based systematic review. Ophthalmology 2007; 114: 835-54.

4. Chen HC, Wiek J, Gupta A, Luckie A, Kohner EM. Effect of isovolaemic haemodilution on visual outcome in branch retinal vein occlusion. Br J Ophthalmol 1998; 82: 162-7.

5. Osterloh MD, Charles S. Surgical decompression of branch retinal vein occlusion. Arch Ophthalmol 1988; 106 : 1469-71.
6. The Branch Vein Occlusion Study Group. Argon laser photocoagulation for macular edema in branch vein occlusion. Am J Ophthalmol 1984; 98: 271-82.

7. Opremcak EM, Bruce RA. Surgical decompression of branch retinal vein occlusion via arteriovenous crossing sheathotomy. Retina 1999; 19: 1-5.

8. Mason J III, Feist R, White M Jr, Swanner J, McGwin G Jr, Emond T. Sheathotomy to decompress branch retinal vein occlusion: a matched control study. Ophthalmology 2004; 111: 540-5.

9. Sohn JH, Song SJ. Arteriovenous sheathotomy for persistent macular edema in branch retinal vein occlusion. Korean J Ophthalmol 2006; 20: 210-4.

10. Figueroa MS, Torres R, Alvarez MT. Comparative study of vitrectomy with and without vein decompression for branch retinal vein occlusion: a pilot study. Eur J Ophthalmol 2004; 14: $40-7$

11. Lee $\mathrm{H}$, Shah GK. Intravitreal triamcinolone as primary treatment of cystoid macular edema secondary to branch retinal 
vein occlusion. Retina 2005; 25: 551-5.

12. Cekiç O, Chang S, Tseng JJ, et al. Intravitreal triamcinolone injection for treatment of macular edema secondary to branch retinal vein occlusion. Retina 2005; 25: 851-5.

13. Salinas-Alamán A, García-Layana A, Sádaba-Echarri LM, Belzunce-Manterola A. Triamcinolona intravítrea en el tratamiento de la oclusión de rama venosa retiniana. Arch Soc Esp Oftalmol 2005; 80: 463-5.

14. Chen SD, Sundaram V, Lochhead J, Patel CK. Intravitreal triamcinolone for the treatment of ischemic macular edema associated with branch retinal vein occlusion. Am J Ophthalmol 2006; 141: 876-83.

15. Jonas JB, Akkoyun I, Kamppeter B, Kreissig I, Degenring RF. Branch retinal vein occlusion treated by intravitreal triamcinolone acetonide. Eye 2005; 19: 65-71.

16. Scott IU, Ip MS, VanVeldhuisen PC, et al, SCORE Study Research Group. A randomized trial comparing the efficacy and safety of intravitreal triamcinolone with standard care to treat vision loss associated with macular edema secondary to branch retinal vein occlusion: the Standard Care vs Corticosteroid for Retinal Vein Occlusion (SCORE) study report 6. Arch Ophthalmol 2009; 127: 1115-28.

17. Parodi $M B$, lacono $P$, Ravalico $G$. Intravitreal triamcinolone acetonide combined with subthreshold grid laser treatment for macular edema in branch retinal vein occlusion: a pilot study. Br J Ophthalmol 2008; 92: 1046-50.

18. Riese J, Loukopoulos V, Meier C, Timmermann M, Gerding $\mathrm{H}$. Combined intravitreal triamcinolone injection and laser photocoagulation in eyes with persistent macular edema after branch retinal vein occlusion. Graefes Arch Clin Exp Ophthalmol 2008; 246: 1671-6.

19. Kuppermann BD, Blumenkranz MS, Haller JA, et al. Randomized controlled study of an intravitreous dexametha- sone drug delivery system in patients with persistent macular edema. Arch Ophthalmol 2007; 125: 309-17.

20. Haller JA, Dugel P, Weinberg DV, Chou C, Whitcup SM. Evaluation of the safety and performance of an applicator for a novel intravitreal dexamethasone drug delivery system for the treatment of macular edema. Retina 2009; 29: 46-51.

21. Haller JA, Bandello F, Belfort R Jr, et al, OZURDEX GENEVA Study Group. Randomized, sham-controlled trial of dexamethasone intravitreal implant in patients with macular edema due to retinal vein occlusion. Ophthalmology 2010; 117: 1134-46.

22. Gutiérrez JC, Barquet LA, Caminal JM, et al. Intravitreal bevacizumab (Avastin) in the treatment of macular edema secondary to retinal vein occlusion. Clin Ophthalmol 2008; 2: 787-91.

23. Gregori NZ, Rattan GH, Rosenfeld PJ, et al. Safety and efficacy of intravitreal bevacizumab (Avastin) for the management of branch and hemiretinal vein occlusion. Retina 2009; 29: 913-25.

24. Ach T, Hoeh AE, Schaal KB, Scheuerle AF, Dithmar S. Predictive factors for changes in macular edema in intravitreal bevacizumab therapy of retinal vein occlusion. Graefes Arch Clin Exp Ophthalmol 2010; 248: 155-9.

25. Wroblewski JJ, Wells JA III, Gonzales CR. Pegaptanib sodium for macular edema secondary to branch retinal vein occlusion. Am J Ophthalmol 2010; 149: 147-54.

26. Campochiaro PA, Hafiz G, Shah SM, et al. Ranibizumab for macular edema due to retinal vein occlusions: implication of VEGF as a critical stimulator. Mol Ther 2008; 16: 791-9.

27. Campochiaro PA, Heier JS, Feiner L, et al, BRAVO Investigators. Ranibizumab for macular edema following branch retinal vein occlusion: six-month primary end point results of a phase III study. Ophthalmology 2010; 117: 1102-12.

\section{an line}

This paper has been selected to appear on the

EJOWEB page free of charge

www.eur-j-ophthalmol.com 